Pacific

Journal of

Mathematics

THE INTEGRAL KERNEL IN THE

KUZNETSOV SUM FORMULA FOR SU $(n+1,1)$ (II).

THE CASE OF ONE DIMENSIONAL $K$-TYPES

N.P. KISBYE

Volume 213 No. 2

February 2004 


\title{
THE INTEGRAL KERNEL IN THE KUZNETSOV SUM FORMULA FOR SU $(n+1,1)$ (II). THE CASE OF ONE DIMENSIONAL $K$-TYPES
}

\author{
N.P. KISBYE
}

\begin{abstract}
The Kuznetsov sum formula relates spectral data concerning automorphic forms to geometric data concerning the intersection of a discrete subgroup with the big cell in the Bruhat decomposition. An explicit formula for the integral kernel in the Kloosterman term of this formula is given for the groups isomorphic to $\mathrm{SU}(n+1,1), n \geq 2$ and arbitrary one dimensional $K$-types.
\end{abstract}

\section{Introduction.}

Let $G$ be a connected semisimple Lie group of real rank one, and let $\Gamma$ be a discrete subgroup of finite covolume of $G$. There is a Kuznetsov formula in this context (see $[\mathbf{M W}]$ ) that relates spectral data concerning automorphic forms to geometric data concerning the intersection of a discrete subgroup with the big cell in the Bruhat decomposition. The $\tau$-function is the kernel for the integral transformation relating test functions on the spectral side to those on the geometric side. In the classical case, this integral transformation can be described in terms of classical Bessel functions (see $[\mathbf{K}]$, $[\mathbf{G W}],[\mathbf{M W}]$, Appendix), but in the general case the determination of the Kloosterman term in the Kuznetsov formula is much more complicated.

This function has been determined when $G=\mathrm{SO}(n+1,1)$ and $\mathrm{SU}(n+1,1)$ (see $[\mathbf{M W}]$ and $[\mathbf{K b}]$ respectively) in the case of the trivial $K$-type. In the present paper we shall extend the methods in $[\mathbf{K b}]$ to obtain a formula for $\tau$ (see $(2.7)$ ) in the case of the group $\mathrm{SU}(n+1,1)$ and an arbitrary one dimensional $K$-type. Similarly as in $[\mathbf{K b}]$ the calculation requires solving complicated recurrence relations.

Let $G=N A K$ be an Iwasawa decomposition of $G$ and let $\mathfrak{g}=\mathfrak{n} \oplus \mathfrak{a} \oplus \mathfrak{k}$ be the corresponding decomposition at the Lie algebra level. Let $M$ be the centralizer of $A$ in $K$ and let $\chi$ be a nontrivial unitary character on $N$. As in $[\mathbf{K b}]$, one necessary ingredient in our computations is the knowledge of generators for the $M_{\chi}$-invariants in the universal Lie algebra $\mathcal{U}(\overline{\mathfrak{n}})$ for those groups (see $[\mathbf{M V}]$ ). The main difference comes from the fact that in this case the recurrence formulas needed to compute the $\tau$-function involve new and complicated terms that become zero only when the $K$-type is trivial. 
However, the final formula for $\tau$ in the present case ends up being similar to that obtained in $[\mathbf{K b}]$, but involving a real parameter that depends on the $K$-type.

A new feature of this case is the presence of poles in the right half plane of the form $\mu-2, \mu-4, \ldots$, where $\mu \in \mathbb{N}$ is the $K$-type parameter.

\section{Preliminaries.}

We consider the Lie subalgebra of $\mathfrak{g l}(n+2, \mathbf{C})$ given by

$$
\mathfrak{g}=\left\{X \in \mathfrak{g l}(n+2, \mathbf{C}) \mid X J+J \bar{X}^{t}=0, \operatorname{tr} X=0\right\}
$$

where

$$
J=\left(\begin{array}{ccc}
0 & 0 & 1 \\
0 & I_{n} & 0 \\
1 & 0 & 0
\end{array}\right)
$$

and $I_{n}$ is the $n \times n$ identity matrix. Then $\mathfrak{g}$ is the Lie algebra $\simeq \mathcal{S U}(n+1,1)$. We denote by $G \simeq \mathrm{SU}(n+1,1)$ the connected Lie subgroup of $\operatorname{Gl}(n+2, \mathbf{C})$ with Lie algebra $\mathfrak{g}$. A Cartan involution of $\mathfrak{g}$ is given by $\theta(X)=-\bar{X}^{t}$.

This involution induces the Cartan decomposition $\mathfrak{g}=\mathfrak{k} \oplus \mathfrak{p}$. We take $\mathfrak{a}$ the maximal abelian subalgebra of $\mathfrak{p}$ given by $\mathfrak{a}=\mathbb{R} H$, where $H=$ $\left(\begin{array}{ccc}1 & 0 & 0 \\ 0 & 0_{n} & 0 \\ 0 & 0 & -1\end{array}\right)$. Let $K$ and $A$ be the connected Lie subgroups of $G$ corresponding to $\mathfrak{k}$ and $\mathfrak{a}$, respectively. Let $M$ be the centralizer of $A$ in $K$, and let $\mathfrak{m}$ be the corresponding Lie algebra of $M$. If $\alpha \in \mathfrak{a}^{*}$ is such that $\alpha(H)=1$, then let $\mathfrak{n}_{\alpha}$ and $\mathfrak{n}_{2 \alpha}$ be the root spaces associated to $\alpha$ and $2 \alpha$, respectively. Let $\rho\left(H_{0}\right)=\left.\frac{1}{2} \operatorname{tr} \operatorname{ad}\left(H_{0}\right)\right|_{\mathfrak{n}}, H_{0} \in \mathfrak{a}$. Then $\rho(H)=n+1$. We have $\mathfrak{n}_{\alpha}=\left\{X(x) \mid x \in \mathbb{C}^{n}\right\}$ and $\mathfrak{n}_{2 \alpha}=\mathbb{R} Z(i)$, where

$$
X(x)=\left(\begin{array}{ccc}
0 & x & 0 \\
0 & 0 & -\bar{x}^{t} \\
0 & 0 & 0
\end{array}\right), \quad Z(i)=\left(\begin{array}{ccc}
0 & 0 & i \\
0 & 0 & 0 \\
0 & 0 & 0
\end{array}\right) .
$$

We also have

$\mathfrak{m}=\left\{M(A)=\left(\begin{array}{ccc}a & \ldots & 0 \\ . & A & . \\ 0 & \ldots & a\end{array}\right) \mid A \in M_{n}(\mathbb{C}), A+\bar{A}^{t}=0,2 a+\operatorname{tr}(A)=0\right\}$.

If $\mathfrak{n}=\mathfrak{n}_{\alpha} \oplus \mathfrak{n}_{2 \alpha}$, then $\mathfrak{g}$ has the Iwasawa decomposition $\mathfrak{g}=\mathfrak{n} \oplus \mathfrak{a} \oplus \mathfrak{k}$. Let $G=N A K$ be the corresponding Iwasawa decomposition at the group level. If $\overline{\mathfrak{n}}=\theta \mathfrak{n}$, the $\mathfrak{g}=\mathfrak{n} \oplus \mathfrak{a} \oplus \mathfrak{m} \oplus \overline{\mathfrak{n}}$.

Let $B(X, Y)=\frac{1}{2} \operatorname{tr}(X . Y),\langle X, Y\rangle=-B(X, \theta Y), X, Y \in \mathfrak{g}$. Then $B$ is $\mathfrak{g}$-invariant and $B(H, H)=1$.

If $e_{1}, \ldots, e_{n}$ denotes the canonical basis in $\mathbb{R}^{n}$, we set $X_{j}=X\left(e_{j}\right)$ and $X^{\prime}{ }_{j}=X\left(i e_{j}\right), Y_{j}=-\theta X_{j}, Y^{\prime}{ }_{j}=-\theta X^{\prime}{ }_{j}, Z=Z(i), Z^{\prime}=-\theta Z$. Then 
$\left\{X_{j}, X^{\prime}{ }_{k}, \sqrt{2} Z \mid 1 \leq j, k \leq n\right\}$ is an orthonormal basis of $\mathfrak{n}$ with respect to $\langle$,$\rangle . Note that \left[X_{i}, X^{\prime}{ }_{j}\right]=2 \delta_{i j} Z$.

If $\chi$ is a character of $N$, then there exists $X_{\chi} \in \mathfrak{n}_{\alpha}$ such that $d \chi(X)=$ $i\left\langle X, X_{\chi}\right\rangle=-i B\left(X, \theta X_{\chi}\right)$, for $X \in \mathfrak{n}$. Set $M_{\chi}=\left\{m \in M \mid \operatorname{Ad}(u) X_{\chi}=X_{\chi}\right\}$. As $M$ acts transitively on the unit sphere of $\mathfrak{n}_{\alpha}$ (cf. [MV, Introduction]) there is $u_{0} \in M$ such that $\operatorname{Ad}\left(u_{0}\right) X_{\chi}=c X_{1}, c \in \mathbb{R}^{+}$. So $M_{\chi}=u_{0} M_{1} u_{0}{ }^{-1}$, where $M_{1}=\left\{u \in M \mid \operatorname{Ad}(u) X_{1}=X_{1}\right\}$.

The compact subgroup $K$ of $G$ is given by the set of matrices of the form $k=k(\theta)=\left(\begin{array}{cc}U & 0 \\ 0 & e^{i \theta}\end{array}\right)$, with $U \in U(n), \operatorname{det}(U)=e^{-i \theta}$. If $\mu \in \mathbb{Z}$ define $\phi_{\mu}(k)=\operatorname{det}(U)^{\mu}=e^{-i \mu \theta}$ and let

$$
\xi_{\mu}=\left.\phi_{\mu}\right|_{M}
$$

Now consider de Verma module $M(-\nu)=\mathcal{U}(\mathfrak{g}) \otimes_{\mathcal{U}(\mathfrak{p})} \mathbb{C}_{-\nu-\rho}$, where $\mathfrak{p}=$ $\mathfrak{m} \oplus \mathfrak{a} \oplus \mathfrak{n}$ and $\mathbb{C}_{-\nu-\rho}$ denotes the $\mathfrak{p}$-module $\mathbb{C}$ with $\mathfrak{m}$ acting by $d \xi_{\mu}, \mathfrak{n}$ acting by 0 and $\mathfrak{a}$ acting by $-\nu-\rho, \nu \in \mathfrak{a}_{c}{ }^{*}$. Let $M(-\nu)[\overline{\mathfrak{n}}]$ denote the $\overline{\mathfrak{n}}$-completion of $M(-\nu)$ (see $[\mathbf{G W}], \S 2)$. If $J=\left(j_{1}, j_{2}, \ldots, j_{m}\right) \in \mathbb{N}^{m},(\mathbb{N}=\{0,1,2, \ldots\})$, $m=2 n+1$, and $Y(J)=Y_{1}^{j_{1}} \ldots Y_{n}{ }^{\prime j_{2 n}} Z^{\prime j_{m}}$, then by Poincaré-Birkhoff-Witt theorem, the set $\left\{Y(J) \mid J \in \mathbb{N}^{m}\right\}$ constitutes a basis of $\mathcal{U}(\overline{\mathfrak{n}})$. Hence every element in $M(-\nu)[\overline{\mathfrak{n}}]$ has an expansion of the type $\sum_{J} a_{J} Y(J) \otimes 1, a_{J} \in \mathbb{C}$. A $\chi$-Whittaker vector is an element $v_{\chi}(-\nu)$ in $M(-\nu)[\overline{\mathfrak{n}}]$ that satisfies the equation

$$
X . v=d \chi(X) v \quad \forall X \in \mathfrak{n} .
$$

Such a vector has an expression of the form

$$
v_{\chi}(-\nu)=\sum_{I \in \mathbb{N}^{m}} a_{I}(\chi,-\nu) Y(I) \otimes 1
$$

where the coefficients $a_{I}(\chi,-\nu) \in \mathbb{C}$ are rational functions of $\nu$. There is a unique such Whittaker vector with $a_{0}(\chi,-\nu)=1$ (see $[\mathbf{B M}] \S 6$, Lemma 11, for instance). Since $m \cdot v_{\chi}(-\nu)=v_{\chi}(-\nu)$ for $m \in M_{\chi}$, we have that for each $I Y(I)$ must be a polynomial in the $M_{\chi}$-invariants of $\mathcal{U}(\overline{\mathfrak{n}})$.

Let $\chi$ be such that

$$
d \chi\left(X_{1}\right)=\lambda, \quad d \chi\left(X_{i}\right)=d \chi\left(X_{j}{ }^{\prime}\right)=0 \quad i>1, j \geq 1,
$$

$\lambda \in i \mathbb{R}$. For this choice of $\chi$, we shall use the notation $u(\lambda,-\nu)$ for the unique $\chi$-Whittaker vector in $M(-\nu)[\overline{\mathfrak{n}}]$ such that $a_{0}(\chi,-\nu)=1$. The coefficients of this $\chi$-Whittaker vector will be denoted $a(\lambda,-\nu)$. Then $u(\lambda,-\nu)$ must be an element in $\mathcal{U}(\overline{\mathfrak{n}})^{M_{1}}$. We note that $M_{1} \simeq \mathrm{SU}(n-1)$ is the subgroup of matrices in $M$ of the form

$$
u_{1}(b, B)=\left(\begin{array}{llll}
b & 0 & 0 & 0 \\
0 & b & 0 & 0 \\
0 & 0 & B & 0 \\
0 & 0 & 0 & b
\end{array}\right),
$$


$B \in \mathrm{U}(n-1)$. Hence $u(\lambda,-\nu)=\sum_{j} Y_{1}^{j} f_{j}$, where $f_{j}$ is an $M_{1}$-invariant polynomial in $Y_{2}, \ldots, Y_{n}, Y_{1}^{\prime}, \ldots, Y_{n}^{\prime}$ and $Z^{\prime}$. Then, by [MV], Theorem $\mathrm{B}, f_{j} \in \mathbb{C}\left[Y_{1}^{\prime}, Z^{\prime}, q_{1}\right]$, where $q_{1}=\sum_{i=2}^{n} Y_{i}^{2}+{Y_{i}^{\prime}}^{2}$.

Let $E_{i, j}$ be the matrix in $\mathfrak{g l}(n+2, \mathbb{C})$ having the entry $(i, j)$ equal to 1 , and all the other entries zero. In order to have simpler formulas, it is convenient to change the basis of $\overline{\mathfrak{n}}_{\mathbb{C}}$ to

$$
\left\{V_{1}, V_{2}, Y_{2}, \ldots, Y_{n}, Y_{2}{ }^{\prime}, \ldots, Y_{n}{ }^{\prime}, T\right\},
$$

where $V_{1}=E_{2,1}, V_{2}=E_{n+2,2}, T=E_{n+2,1}$. Note that $Y_{1}=V_{1}-V_{2}$, $Y_{1}^{\prime}=-i V_{1}-i V_{2}$, and $Z^{\prime}=-i \frac{1}{\sqrt{2}} T$. Let $q$ be the element in $\mathcal{U}(\overline{\mathfrak{n}})^{M_{1}}$,

$$
q=\sum_{i=1}^{n} Y_{i}^{2}+Y_{i}^{\prime 2} .
$$

Now it is clear that we may write

$$
\begin{aligned}
& u(\lambda,-\nu)=\sum_{j, k, l \geq 0} a_{j, k, l}(\lambda,-\nu) V_{1}{ }^{j} V_{2}{ }^{k} T^{l} \otimes 1 \quad n=1 \\
& u(\lambda,-\nu)=\sum_{j, k, l, m \geq 0} a_{j, k, l, m}(\lambda,-\nu) V_{1}{ }^{j} V_{2}{ }^{k} T^{l} q^{m} \otimes 1 \quad n>1 .
\end{aligned}
$$

Now we use the $T(\nu)$-transform $([\mathbf{G W}]$ and $[\mathbf{M W} \mathbf{1}])$ to compute the $\tau$ function. We see in $[\mathbf{M W}]$ that this $\tau$-function is the main ingredient in the Bessel transform in the Kloosterman term of the sum formula of Kuznetsov type and also it appears in the Fourier coefficients of the Poincaré series. To give the formula of the $\tau$-function we consider two parabolic $\Gamma$-percuspidal subgroups $P$ and $P^{\prime}$. Then $P=N A M$ and $P^{\prime}=N^{\prime} A^{\prime} M^{\prime}$. Let $\chi$ and $\chi_{1}$ be nontrivial unitary characters on $N$ and $N^{\prime}$ respectively. If $W(A)=\{1, s\}$ is the Weyl group of $(P, A)$ then we take $s^{*}$ a representative of $s$ in $K$. The $\tau$-function is given by the following formula (see [MW] Proposition 1.2):

$$
\tau\left(\chi_{1}, \chi, u a, \nu\right)=\sum_{I \in \mathbb{N}^{m}} a_{I}(\chi, \nu) d \chi_{1}\left(\operatorname{Ad}\left(u a s^{*}\right)^{-1} Y(I)^{T}\right) \otimes 1
$$

$u \in M, a \in A$ and where the coefficients $a_{I}$ are given by Formula (2.3). Here, $Y \mapsto Y^{T}$ is the automorphism of the universal enveloping algebra given by $X \mapsto-X$, for $X \in \mathfrak{g}$.

\section{An explicit formula for the $\chi^{-W h i t t a k e r ~ v e c t o r . ~}$}

The aim of this section is to give a formula for a Whittaker vector $u(\lambda, \nu+\rho)$ in the $\overline{\mathfrak{n}}$-completion of the Verma module $M(\nu+\rho)=\mathcal{U}(\mathfrak{g}) \otimes_{\mathcal{U}} \mathbb{C}_{\nu}$, where $\mathfrak{m}$ acts on $\mathbb{C}_{\nu}$ by $d \xi_{\mu}$ with $\xi_{\mu}$ as in (2.1), $\mathfrak{n}$ acts by 0 and $\mathfrak{a}$ acts by $\nu, \nu \in \mathfrak{a}^{*}$. In what follows we shall write $\nu$ instead of $\nu(H)$. 
Lemma 3.1. Let $X_{1}, X_{1}{ }^{\prime}, V_{1}, V_{2}, T$ and $H$ be as above. Let $U^{+}=$ $\frac{1}{2}\left[H+i M\left(2 i E_{11}\right)\right]$ and $U^{-}=\frac{1}{2}\left[-H+i M\left(2 i E_{11}\right)\right]$. Then, the following commutation relations hold for $j, k, l \geq 1$ :

$$
\begin{aligned}
{\left[X_{1}, V_{1}{ }^{j}\right] } & =j V_{1}^{j-1}\left(U^{+}-j+1\right) \\
{\left[X_{1}, V_{2}{ }^{k}\right] } & =k V_{2}{ }^{k-1}\left(U^{-}+k-1\right) \\
{\left[X_{1}, T^{l}\right] } & =-l\left(V_{1}+V_{2}\right) T^{l-1} \\
{\left[X^{\prime}{ }_{1}, V_{1}{ }^{j}\right] } & =j V_{1}{ }^{j-1}\left(i U^{+}-i(j-1)\right) \\
{\left[X_{1}{ }^{\prime}, V_{2}{ }^{k}\right] } & =k V_{2}{ }^{k-1}\left(-i U^{-}-i(k-1)\right) \\
{\left[X_{1}{ }^{\prime}, T^{l}\right] } & =i l\left(V_{1}-V_{2}\right) T^{l-1} .
\end{aligned}
$$

Also we have: $\left[U^{+}, V_{2}\right]=V_{2},\left[U^{+}, T\right]=-T$ and $\left[U^{-}, T\right]=T$.

Proof. The formulas are proved by the usual $\mathrm{Sl}(2, \mathbb{C})$-technique. They are based on the identities: $\left[X_{1}, V_{1}\right]=U^{+},\left[X_{1}, V_{2}\right]=U^{-},\left[X_{1}{ }^{\prime}, V_{1}\right]=i U^{+}$, $\left[X_{1}{ }^{\prime}, V_{2}\right]=-i U^{-}$and on the fact that $\left[V_{1}, V_{2}\right]=-T$.

Thus, it follows from Lemma 3.1 that:

$$
\begin{aligned}
& X_{1} \cdot V_{1}^{j} V_{2}{ }^{k} T^{l} \otimes 1 \\
&=\left(X_{1} \cdot V_{1}^{j}\right) V_{2}^{k} T^{l} \otimes 1+V_{1}^{j}\left(X_{1} \cdot V_{2}{ }^{k}\right) T^{l} \otimes 1+V_{1}^{j} V_{2}{ }^{k}\left(X_{1} \cdot T^{l}\right) \otimes 1 \\
&= j V_{1}^{j-1}\left(U^{+}-j+1\right) V_{2}{ }^{l} T^{l} \otimes 1+k V_{1}^{j} V_{2}{ }^{k-1}\left(U^{-}+k-1\right) T^{l} \otimes 1 \\
&-l V_{1}^{j} V_{2}{ }^{k}\left(V_{1}+V_{2}\right) T^{l-1} \otimes 1 \\
&= j V_{1}^{j-1} V_{2}{ }^{k} T^{l}\left(k-l-j+1+U^{+}\right) \otimes 1 \\
&+k V_{1}^{j} V_{2}{ }^{k-1} T^{l}\left(l+k-1+U^{-}\right) \otimes 1 \\
&-l V_{1}{ }^{j} V_{2}{ }^{k} V_{1} T^{l-1} \otimes 1-l V_{1}{ }^{j} V_{2}{ }^{k+1} T^{l-1} \otimes 1 .
\end{aligned}
$$

Using that $\left[V_{1}, V_{2}{ }^{k}\right]=-k V_{2}{ }^{k-1} T$ and the fact that $U^{+}$and $U^{-}$act on $\mathbb{C}_{\nu}$ by $\frac{\nu+\mu}{2}$ and $\frac{-\nu+\mu}{2}$ respectively, the last expression is equal to

$$
\begin{aligned}
& j V_{1}^{j-1} V_{2}{ }^{k} T^{l}\left(k-l-j+1+\frac{\nu+\mu}{2}\right) \otimes 1 \\
& +k V_{1}^{j} V_{2}{ }^{k-1} T^{l}\left(k-1-\frac{\nu-\mu}{2}\right) \otimes 1 \\
& \quad-l V_{1}^{j+1} V_{2}{ }^{k} T^{l-1} \otimes 1-l V_{1}^{j} V_{2}{ }^{k+1} T^{l-1} \otimes 1 .
\end{aligned}
$$


Now, Formula (2.2) implies that:

$$
\begin{aligned}
& \sum_{j, k, l \geq 0} a_{j, k, l}(\lambda, \nu+\rho) X_{1} \cdot V_{1}{ }^{j} V_{2}{ }^{k} T^{l} \otimes 1 \\
& =\lambda \sum_{j, k, l \geq 0} a_{j, k, l}(\lambda, \nu+\rho) V_{1}{ }^{j} V_{2}{ }^{k} T^{l} \otimes 1, \\
& \sum_{j, k, l \geq 0} a_{j, k, l}(\lambda, \nu+\rho) X_{1}{ }^{\prime} \cdot V_{1}{ }^{j} V_{2}{ }^{k} T^{l} \otimes 1=0 .
\end{aligned}
$$

From Formulas (3.2) and (3.3) we obtain the following recurrence relation for the coefficients $a_{j, k, l}(\lambda, \nu+\rho)$ :

$$
\begin{aligned}
& \lambda a_{j, k, l}(\lambda, \nu+\rho) \\
& =(j+1)\left(k-l-j+\frac{\nu+\mu}{2}\right) a_{j+1, k, l}(\lambda, \nu+\rho) \\
& \quad+(k+1)\left(k-\frac{\nu-\mu}{2}\right) a_{j, k+1, l}(\lambda, \nu+\rho) \\
& \quad-(l+1) a_{j-1, k, l+1}(\lambda, \nu+\rho)-(l+1) a_{j, k-1, l+1}(\lambda, \nu+\rho) .
\end{aligned}
$$

Also, we have

$$
\begin{aligned}
X_{1}{ }^{\prime} & \cdot V_{1}{ }^{j} V_{2}{ }^{k} T^{l} \otimes 1 \\
= & \left(X_{1}{ }^{\prime} \cdot V_{1}{ }^{j}\right) V_{2}{ }^{k} T^{l} \otimes 1+V_{1}{ }^{j}\left(X_{1}{ }^{\prime} \cdot V_{2}{ }^{k}\right) T^{l} \otimes 1+V_{1}{ }^{j} V_{2}{ }^{k}\left(X_{1}{ }^{\prime} \cdot T^{l}\right) \otimes 1 \\
= & i j V_{1}{ }^{j-1}\left(U^{+}-j+1\right) V_{2}{ }^{k} T^{l} \otimes 1+V_{1}{ }^{l} k V_{2}{ }^{-1}\left(U^{-}-i(k-1)\right) T^{l} \otimes 1 \\
& +V_{1}{ }^{j} V_{2}{ }^{k}\left(i l\left(V_{1}-V_{2}\right)\right) T^{l-1} \otimes 1 \\
= & i j V_{1}{ }^{j-1} V_{2}{ }^{k} T^{l}\left(U^{+}-j+1+k-l\right) \otimes 1 \\
& +V_{1}{ }^{j} k V_{2}{ }^{k-1} T^{l}\left(U^{-}-i(k-1+l)\right) \otimes 1 \\
& +i l V_{1}{ }^{j} V_{2}{ }^{k} V_{1} T^{l-1} \otimes 1-i l V_{1}{ }^{j} V_{2}{ }^{k+1} T^{l-1} \otimes 1 \\
= & i j V_{1}{ }^{j-1} V_{2}{ }^{k} T^{l}\left(-j+1+k-l+\frac{\nu+\mu}{2}\right) \otimes 1 \\
& +i V_{1}{ }^{j} k V_{2}{ }^{k-1} T^{l}\left(-k+1+\frac{\nu-\mu}{2}\right) \otimes 1 \\
& +i l V_{1}{ }^{j+1} V_{2}{ }^{k} T^{l-1} \otimes 1-i l V_{1}{ }^{j} V_{2}{ }^{k+1} T^{l-1} \otimes 1 .
\end{aligned}
$$


This computation together with Formula (3.4) gives a second recurrence relation for the coefficients $a_{j, k, l}(\lambda, \nu+\rho)$ :

$$
\begin{aligned}
& (j+1)\left(-j+k-l+\frac{\nu+\mu}{2}\right) a_{j+1, k, l}(\lambda, \nu+\rho) \\
& \quad+(k+1)\left(-k+\frac{\nu-\mu}{2}\right) a_{j, k+1, l}(\lambda, \nu+\rho) \\
& \quad+(l+1) a_{j-1, k, l+1}(\lambda, \nu+\rho)-(l+1) a_{j, k-1, l+1(\lambda, \nu+\rho)}=0
\end{aligned}
$$

Finally, from the identities (3.5) and (3.7) we get the following relations:

$$
\begin{aligned}
\lambda a_{j, k, l}(\lambda, \nu+\rho)= & (j+1)(\nu+\mu-2 j+2 k-2 l) a_{j+1, k, l}(\lambda, \nu+\rho) \\
& -2(l+1) a_{j, k-1, l+1}(\lambda, \nu+\rho), \\
\lambda a_{j, k, l}(\lambda, \nu+\rho)= & (k+1)(-\nu+\mu+2 k) a_{j, k+1, l}(\lambda, \nu+\rho) \\
& -2(l+1) a_{j-1, k, l+1}(\lambda, \nu+\rho) .
\end{aligned}
$$

Proposition 3.2. Let $\chi$ be as in (2.4). If $u(\lambda, \nu+\rho)$ denotes the canonical $\chi$-Whittaker vector in $M(\nu+\rho)$ for $\mathrm{SU}(2,1)$, then the coefficients $a_{j, k, l}(\lambda, \nu+$ $\rho)$ of $u(\lambda, \nu+\rho)$ are given by the formula:

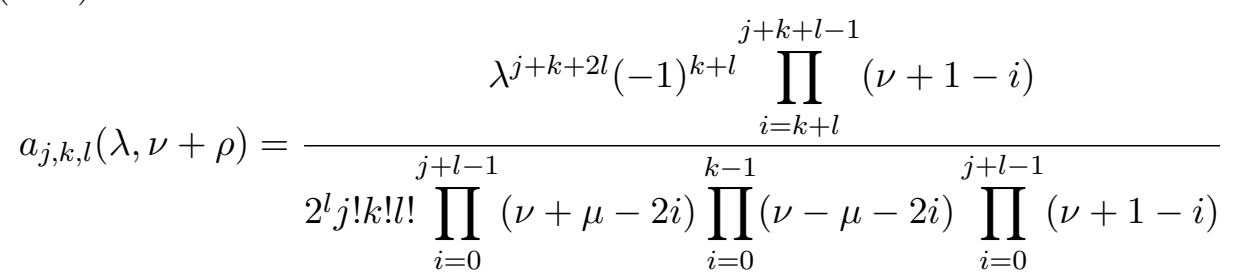

$a_{0,0,0}(\lambda, \nu+\rho)=1$.

The proof of this proposition follows by induction on $n=j+k+2 l$ and is very similar to the proof of Proposition 3.2 in $[\mathbf{K b}]$.

Now we shall compute the coefficients of the $\chi$-Whittaker vector for $\mathrm{SU}(n+1,1)$ in the case $n>1$. We set

$$
M_{j k}=M\left(E_{j k}-E_{k j}\right), \quad M^{j k}=M\left(i E_{j k}+i E_{k j}\right) .
$$

Then $d \xi\left(M_{i j}\right)=0$ and $d \xi\left(M^{i j}\right)=\delta_{i j}(-i \mu)$. 
Lemma 3.3. Let $X_{i}, X_{i}^{\prime}, Y_{i}, Y_{i}^{\prime}, V_{1}, V_{2}, T$ and $q$ be as in Section 1. Then the following identities hold: For $i \geq 2$,

$$
\begin{aligned}
{\left[X_{i}, V_{1}^{j}\right] } & =j V_{1}^{j-1} M_{i} \quad \text { where } M_{i}=\frac{1}{2}\left(M\left(E_{i 1}-E_{1 i}\right)+i M\left(i E_{i 1}+i E_{1 i}\right)\right) \\
{\left[X_{i}, V_{2}^{k}\right] } & =k V_{2}^{k-1}\left(M_{i}\right)^{t} \\
{\left[X_{i}, T^{l}\right] } & =-i l T^{l-1}\left(Y_{i}^{\prime}\right) \\
{\left[X_{i}^{\prime}, V_{1}^{j}\right] } & =i j V_{1}^{j-1} M_{i} \\
{\left[X_{i}^{\prime}, V_{2}^{k}\right] } & =-i k V_{2}^{k-1}\left(M_{i}\right)^{t} \\
{\left[X_{i}^{\prime}, T^{l}\right] } & =i l T^{l-1}\left(Y_{i}^{\prime}\right) \\
{\left[M_{i}, V_{2}^{k}\right] } & =(k / 2)\left(-Y_{i}+i Y_{i}^{\prime}\right) V_{2}^{k-1} .
\end{aligned}
$$

For $i \geq 1$,

(3.12) $\left[X_{i}, q^{m}\right]$

$$
\begin{aligned}
= & 2 \sum_{s=0}^{m-1} 4^{s} U_{i}^{(s)} T^{s} q^{m-1-s} \sum_{j=s}^{m-1}\left(\begin{array}{l}
j \\
s
\end{array}\right)(n+H-2(m-1-j)) \\
& +2 \sum_{s=0}^{m-1} 4^{s}\left(\begin{array}{c}
m \\
s+1
\end{array}\right) T^{s} q^{m-1-s} \Phi^{(s)}\left(X_{i}\right),
\end{aligned}
$$

(3.13) $\left[X_{i}^{\prime}, q^{m}\right]$

$$
\begin{aligned}
= & 2 i \sum_{s=0}^{m-1} 4^{s} U_{i}^{(s-1)} T^{s} q^{m-1-s} \sum_{j=s}^{m-1}\left(\begin{array}{l}
j \\
s
\end{array}\right)(n+H-2(m-1-j)) \\
& -2 \sum_{s=0}^{m-1} 4^{s}\left(\begin{array}{c}
m \\
s+1
\end{array}\right) T^{s} q^{m-1-s} \Phi^{(s)}\left(X_{i}^{\prime}\right)
\end{aligned}
$$

where $U_{k}^{(s)}=Y_{k}$ if $s$ is even and $-i Y_{k}^{\prime}$ if $s$ is odd. $\Phi^{(s)}\left(X_{i}\right)$ and $\Phi^{(s)}\left(X_{1}^{\prime}\right)$ belong to $\mathcal{U}(\mathfrak{g}) \mathfrak{m}$ and are given by the formulas

$$
\Phi^{(s)}\left(X_{i}\right)=\left\{\begin{array}{l}
\sum_{j=1}^{n}\left(Y_{j} M_{i j}+Y_{j}^{\prime} M^{i j}\right), \text { if } s \text { is even, } \\
i \sum_{j=1}^{n}\left(Y_{j}^{\prime} M_{i j}-Y_{j} M^{i j}\right), \quad \text { if } s \text { is odd, }
\end{array}\right.
$$

and

$$
\Phi^{(s)}\left(X_{i}^{\prime}\right)=\left\{\begin{array}{l}
\sum_{j=1}^{n}\left(Y_{j} M^{i j}+Y_{j}^{\prime} M_{i j}\right), \text { if } s \text { is even }, \\
i \sum_{j=1}^{n}\left(Y_{j}^{\prime} M^{i j}-Y_{j} M_{i j}\right), \text { if } s \text { is odd. }
\end{array}\right.
$$


Proof. For the first formulas see $[\mathbf{K b}]$, Lemma 3.1. To prove Formula (3.12) we proceed by induction on $m$. The proof of (3.13) is similar. We need the formulas:

$$
\begin{aligned}
{\left[\left[X_{i}, Y_{j}\right], Y_{k}\right] } & =-\delta_{i j} Y_{k}+\delta_{j k} Y_{i}-\delta_{i k} Y_{j} \\
{\left[\left[X_{i}, Y_{j}^{\prime}\right], Y_{k}^{\prime}\right] } & =\delta_{i j} Y_{k}+\delta_{j k} Y_{i}+\delta_{i k} Y_{j} \\
{\left[X_{i}, Y_{j}\right] } & =\delta_{i j} H+M\left(E_{i j}-E_{j i}\right) \\
{\left[X_{i}, Y_{j}^{\prime}\right] } & =M\left(i\left(E_{i j}+E_{j i}\right)\right) \\
{\left[X_{i}^{\prime}, Y_{j}\right] } & =-M\left(i\left(E_{i j}+E_{j i}\right)\right) \\
{\left[X_{i}^{\prime}, Y_{j}^{\prime}\right] } & =\delta_{i j} H+M\left(E_{i j}-E_{j i}\right) \\
{\left[\left[X_{i}^{\prime}, Y_{j}\right], Y_{k}\right] } & =\delta_{i j} Y_{k}^{\prime}+\delta_{j k} Y_{i}^{\prime}+\delta_{i k} Y_{j}^{\prime} \\
{\left[\left[X_{i}^{\prime}, Y_{j}^{\prime}\right], Y_{j}^{\prime}\right] } & =-\delta_{i j} Y_{k}^{\prime}+\delta_{j k} Y_{i}^{\prime}-\delta_{i k} Y_{j}^{\prime} .
\end{aligned}
$$

For $m=1$ we have

$$
\begin{aligned}
{\left[X_{i}, q\right] } & =\sum_{j=1}^{n}\left(\left[X_{i}, Y_{j}^{2}\right]+\left[X_{i}, Y_{j}^{\prime 2}\right]\right) \\
& =\sum_{j=1}^{n}\left(\left[\left[X_{i}, Y_{j}\right], Y_{j}\right]+\left[\left[X_{i}, Y_{j}^{\prime}\right], Y_{j}^{\prime}\right]+2 Y_{j}\left[X_{i}, Y_{j}\right]+2 Y_{j}^{\prime}\left[X_{i}, Y_{j}^{\prime}\right]\right) \\
& =\sum_{j=1}^{n}\left(-2 \delta_{i j} Y_{j}+Y_{i}+2 \delta_{i j} Y_{j}+Y_{i}\right)+2 Y_{i} H+2 Y_{j} M_{i j}+2 Y_{j}^{\prime} M^{i j} \\
& =2 Y_{i}(n+H)+2 \sum_{j=1}^{n}\left(Y_{j} M_{i j}+Y_{j}^{\prime} M^{i j}\right) \\
& =2 Y_{i}(n+H)+2 \Phi^{(0)}\left(X_{i}\right) .
\end{aligned}
$$

If $m>1$ we need the following identities:

$$
\sum_{j=s}^{m-1}\left(\begin{array}{l}
j \\
s
\end{array}\right)(n+H-2(m-1-j)) q=q \sum_{j=s}^{m-1}\left(\begin{array}{l}
j \\
s
\end{array}\right)(n+H-2(m-j)),
$$

$$
\begin{aligned}
& {\left[q^{m}, Y_{i}\right]=\sum_{s=1}^{m} 4^{s} U_{i}^{(s)} T^{s} q^{m-s},} \\
& {\left[\Phi^{(s)}, q\right]=4 T \Phi^{(s+1)} .}
\end{aligned}
$$

Formula (3.15) follows from the fact that $[H, q]=-2 q$, and (3.16) was proved in $[\mathbf{K b}]$, Lemma 3.3. To prove (3.17) we observe that if $s$ is even 
then

$$
\begin{aligned}
{\left[\Phi^{(s)}\left(X_{i}\right), q\right] } & =\sum_{j=1}^{n}\left(\left[Y_{j}, q\right] M_{i j}+\left[Y_{j}^{\prime}, q\right] M^{i j}\right) \\
& =4 i T \sum_{j=1}^{n}\left(Y_{j}^{\prime} M_{i j}-Y_{j} M^{i j}\right)=4 T \Phi^{(s+1)}\left(X_{i}\right) .
\end{aligned}
$$

If $s$ is odd then

$$
\begin{aligned}
{\left[\Phi^{(s)}\left(X_{i}\right), q\right] } & =i \sum_{j=1}^{n}\left(\left[Y_{j}^{\prime}, q\right] M_{i j}-\left[Y_{j}, q\right] M^{i j}\right) \\
& =4 T \sum_{j=1}^{n}\left(Y_{j}^{\prime} M_{i j}+Y_{j} M^{i j}\right)=4 T \Phi^{(s+1)}\left(X_{i}\right) .
\end{aligned}
$$

Thus (3.17) follows.

Using that $\left[X_{i}, q^{m+1}\right]=\left[X_{i}, q^{m}\right] q+q^{m}\left[X_{i}, q\right]$ we have

$$
\begin{aligned}
{\left[X_{i}, q^{m+1}\right]=} & 2 \sum_{s=0}^{m-1} 4^{s} U_{i}^{(s)} T^{s} q^{m-s} \sum_{j=s}^{m-1}\left(\begin{array}{l}
j \\
s
\end{array}\right)(n+H-2(m-j)) \\
& +2 q^{m} Y_{i}(n+H)+2 \sum_{s=0}^{m-1} 4^{s}\left(\begin{array}{c}
m \\
s+1
\end{array}\right) T^{s} q^{m-s} \Phi^{(s)}\left(X_{i}\right) \\
& +2 \sum_{s=0}^{m-1} 4^{s+1}\left(\begin{array}{c}
m \\
s+1
\end{array}\right) T^{s+1} q^{m-(s+1)} \Phi^{(s+1)}\left(X_{i}\right)+2 q^{m} \Phi^{(0)}\left(X_{i}\right),
\end{aligned}
$$

thus

$$
\begin{aligned}
& {\left[X_{i}, q^{m+1}\right]} \\
& =2 \sum_{s=0}^{m-1} 4^{s} U_{i}^{(s)} T^{s} q^{m-s} \sum_{j=s}^{m-1}\left(\begin{array}{c}
j \\
s
\end{array}\right)(n+H-2(m-j)) \\
& \quad+2 U_{i}^{(0)} q^{m}(n+H)+2 \sum_{s=1}^{m} 4^{s}\left(\begin{array}{c}
m \\
s
\end{array}\right) U_{i}^{(s)} T^{s} q^{m-s}(n+H) \\
& \quad+2\left(\begin{array}{c}
m \\
1
\end{array}\right) q^{m} \Phi^{(0)}\left(X_{i}\right)+2 \sum_{s=1}^{m-1} 4^{s}\left(\left(\begin{array}{c}
m \\
s+1
\end{array}\right)+\left(\begin{array}{c}
m \\
s
\end{array}\right)\right) T^{s} q^{m-s} \Phi^{(s)}\left(X_{i}\right) \\
& \quad+24^{m}\left(\begin{array}{c}
m \\
m
\end{array}\right) T^{m} \Phi^{(m)}\left(X_{i}\right)+2 q^{m} \Phi^{(0)}\left(X_{i}\right) .
\end{aligned}
$$


Now,

$$
\begin{aligned}
& 2 U_{i}^{(0)} q^{m} \sum_{j=0}^{m-1}\left(\begin{array}{l}
j \\
0
\end{array}\right)(n+H-2(m-j))+2 U_{i}^{(0)} q^{m}(n+H) \\
& =2 U_{i}^{(0)} q^{m} \sum_{j=0}^{m}\left(\begin{array}{l}
j \\
0
\end{array}\right)(n+H-2(m-j))
\end{aligned}
$$

and

$$
\begin{aligned}
& 2 \sum_{s=1}^{m} 4^{s} U_{i}^{(s)} T^{s} q^{m-s} \sum_{j=s}^{m-1}\left(\begin{array}{l}
j \\
s
\end{array}\right)(n+H-2(m-j)) \\
& \quad+2 \sum_{s=1}^{m-1} 4^{s}\left(\begin{array}{c}
m \\
s
\end{array}\right) U_{i}^{(s)} T^{s} q^{m-s}(n+H) \\
& =2 \sum_{s=1}^{m-1} 4^{s} U_{i}^{(s)} T^{s} q^{m-s} \sum_{j=s}^{m}\left(\begin{array}{l}
j \\
s
\end{array}\right)(n+H-2(m-j)) \\
& \quad+24^{m} U_{i}^{(m)} T^{m}(n+H) \\
& =2 \sum_{s=1}^{m} 4^{s} U_{i}^{(s)} T^{s} q^{m-s} \sum_{j=s}^{m}\left(\begin{array}{l}
j \\
s
\end{array}\right)(n+H-2(m-j)) .
\end{aligned}
$$

Using the identity $\left(\begin{array}{c}m \\ s+1\end{array}\right)+\left(\begin{array}{c}m \\ s\end{array}\right)=\left(\begin{array}{c}m+1 \\ s+1\end{array}\right)$ we have that the sum of the last four terms in Formula (3.18) equals

$$
2 \sum_{s=0}^{m} 4^{s}\left(\begin{array}{c}
m+1 \\
s+1
\end{array}\right) T^{s} q^{m-s} \Phi^{(s)}\left(X_{i}\right) .
$$

Thus we obtain

$$
\begin{aligned}
{\left[X_{i}, q^{m+1}\right]=} & 2 \sum_{s=0}^{m} 4^{s} U_{i}^{(s)} T^{s} q^{m-s} \sum_{j=s}^{m}\left(\begin{array}{c}
j \\
s
\end{array}\right)(n+H-2(m-j)) \\
& +2 \sum_{s=0}^{m} 4^{s}\left(\begin{array}{c}
m+1 \\
s+1
\end{array}\right) T^{s} q^{m-s} \Phi^{(s)}\left(X_{i}\right),
\end{aligned}
$$

so the proof of Formula (3.12) is complete. Using similar computations and the identity

$$
\left[q^{m}, Y_{i}^{\prime}\right]=\sum_{s=1}^{m} 4^{s}\left(\begin{array}{c}
m \\
s
\end{array}\right) U_{i}^{(s-1)} T^{s} q^{m-s},
$$

whose proof can also be found in $[\mathbf{K b}]$, Lemma 3.3, one can obtain Formula $(3.13)$. 
Now, it follows that for $i \geq 2$

$$
\begin{aligned}
X_{i} & \cdot V_{1}^{j} V_{2}^{k} T^{l} q^{m} \otimes 1 \\
= & j V_{1}^{j-1} M_{i} V_{2}^{k} T^{l} q^{m} \otimes 1 \\
& +k V_{1}^{j} V_{2}^{k-1} M_{i}^{t} T^{l} q^{m} \otimes 1-i l V_{1}^{j} V_{2}^{k} T^{l-1} Y_{i}^{\prime} q^{m} \otimes 1 \\
& +V_{1}^{j} V_{2}^{k} T^{l} X_{i} \cdot q^{m} \otimes 1 .
\end{aligned}
$$

As $\mathfrak{m}$ acts by $d \xi_{\mu}$ and from the definition of $M_{k l}$ and $M^{k l}$ in (3.11), we have that $M_{i} \otimes 1=M_{i}^{t} \otimes 1=0$ for $i \geq 2$ and

$$
\Phi^{(s)}\left(X_{i}\right) \otimes 1=\left\{\begin{array}{l}
Y_{i}^{\prime} M^{i i} \otimes 1=-i \mu Y_{i}^{\prime}, \text { if } s \text { is even } \\
-i Y_{i} M^{i i} \otimes 1=-\mu Y_{i}, \text { if } s \text { is odd }
\end{array}\right.
$$

Using that $[\mathfrak{m}, T]=[\mathfrak{m}, q]=0$ and setting $\sigma(m-1, s, \nu)=\sum_{j=s}^{m-1}\left(\begin{array}{l}j \\ s\end{array}\right)(n+$ $\nu-2(m-1-j))$ it follows that

$$
\begin{aligned}
X_{i} & \cdot V_{1}^{j} V_{2}^{k} T^{l} q^{m} \otimes 1 \\
= & \frac{j k}{2} V_{1}^{j-1}\left(-Y_{i}+i Y_{i}^{\prime}\right) V_{2}^{k-1} T^{l} q^{m} \otimes 1-i l V_{1}^{j} V_{2}^{k} T^{l-1} Y_{i}^{\prime} q^{m} \otimes 1 \\
& +2 Y_{i} \sum_{s=0, s \text { even }}^{m-1} 4^{s} \sigma(m-1, s, \nu) V_{1}^{j} V_{2}^{k} T^{l+s} q^{m-1-s} \\
& -2 i Y_{i}^{\prime} \sum_{s=0, s \text { odd }}^{m-1} 4^{s} \sigma(m-1, s, \nu) V_{1}^{j} V_{2}^{k} T^{l+s} q^{m-1-s} \\
& -2 i \mu Y_{i}^{\prime} \sum_{s=0, s \text { even }}^{m-1} 4^{s}\left(\begin{array}{c}
m \\
s+1
\end{array}\right) V_{1}^{j} V_{2}^{k} T^{l+s} q^{m-1-s} \\
& -2 \mu Y_{i} \sum_{s=0, s \text { odd }}^{m-1} 4^{s}\left(\begin{array}{c}
m \\
s+1
\end{array}\right) V_{1}^{j} V_{2}^{k} T^{l+s} q^{m-1-s} \\
= & Y_{i}\left(-\frac{j k}{2} V_{1}^{j-1} V_{2}^{k-1} T^{l} q^{m} \otimes 1+2 \sum_{s=0, s \text { even }}^{m-1} 4^{s} \sigma(m-1, s, \nu) V_{1}^{j} V_{2}^{k} T^{l+s} q^{m-1-s}\right. \\
& -2 \mu \sum_{s=0, s \text { odd }}^{m-1} 4^{s}\left(\begin{array}{c}
m \\
s+1
\end{array}\right) V_{1}^{j} V_{2}^{k} T^{l+s} q^{m-1-s} \\
& -i Y_{i}^{\prime}\left(\sum_{-}^{j k} V_{1}^{j-1} V_{2}^{k-1} T^{l} q^{m} \otimes 1+l V_{1}^{j} V_{2}^{k} T^{l-1} q^{m} \otimes 1\right.
\end{aligned}
$$




$$
\begin{gathered}
+2 \sum_{s=0, s \text { odd }}^{m-1} 4^{s} \sigma(m-1, s, \nu) V_{1}^{j} V_{2}^{k} T^{l+s} q^{m-1-s} \\
\left.\cdot \sum_{s=0, s \text { even }}^{m-1} 4^{s}\left(\begin{array}{c}
m \\
s+1
\end{array}\right) V_{1}^{j} V_{2}^{k} T^{l+s} q^{m-1-s}\right) .
\end{gathered}
$$

Using that $X_{i} \cdot u(\lambda, \nu)=0$ for $i \geq 2$ and the linear independence of the monomials $Y_{i} V_{1}^{j} V_{2}^{k} T^{l} q^{m}$ and $Y_{i}^{\prime} V_{1}^{j} V_{2}^{k} T^{l} q^{m}$ we obtain from the last identity two recurrence formulas for the coefficients $a_{j, k, l, m}$ :

$$
\begin{aligned}
& (j+1)(k+1) a_{j+1, k+1, l, m} \\
& =2 \sum_{s=0}^{l} 4^{s} \sigma(m+s, s, \nu) a_{j, k, l-s, m+1+s} \\
& +(l+1) a_{j, k, l+1, m}+2 \mu \sum_{s=0}^{l}(-1)^{s}\left(\begin{array}{c}
m+1+s \\
s+1
\end{array}\right) a_{j, k, l-s, m+1+s} . \\
& (l+1) a_{j, k, l+1, m}=2 \sum_{s=0}^{l}(-1)^{s} 4^{s} \sigma(m+s, s, \nu) a_{j, k, l-s, m+1+s} \\
& -2 \mu \sum_{s=0}^{l} 4^{s}\left(\begin{array}{c}
m+s+1 \\
s+1
\end{array}\right) a_{j, k, l-s, m+1+s} .
\end{aligned}
$$

To compute the action of $X_{1}$ we need the identities

$$
\begin{aligned}
& V_{2}^{k} Y_{1}=k V_{2}^{k-1} T+V_{1} V_{2}^{k}-V_{2}^{k+1} \\
& V_{2}^{k} Y_{1}^{\prime}=-i k V_{2}^{k-1} T+-i V_{1} V_{2}^{k}-i V_{2}^{k+1}
\end{aligned}
$$

and the commutation relations in Lemma 3.1. Thus

$$
\begin{aligned}
X_{1} & \cdot V_{1}^{j} V_{2}^{k} T^{l} q^{m} \otimes 1 \\
= & j\left(\frac{\nu+\mu}{2}+k-l-j-m+1\right) V_{1}^{j-1} V_{2}^{k} T^{l} q^{m} \otimes 1 \\
& +k\left(\frac{\mu-\nu}{2}+k+m-1\right) V_{1}^{j} V_{2}^{k-1} T^{l} q^{m} \otimes 1 \\
& -l V_{1}^{j+1} V_{2}^{k} T^{l-1} q^{m} \otimes 1-l V_{1}^{j} V_{2}^{k+1} T^{l-1} q^{m} \otimes 1 \\
& +2 \sum_{s=0, s \text { even }} 4^{s} \sigma(m-1, s, \nu)\left(k V_{1}^{j} V_{2}^{k-1} T^{l+s+1} q^{m-1-s}\right. \\
& \left.+V_{1}^{j+1} V_{2}^{k} T^{l+s} q^{m-1-s}-V_{1}^{j} V_{2}^{k+1} T^{l+s} q^{m-1-s}\right)
\end{aligned}
$$




$$
\begin{aligned}
& -2 \sum_{s=0, s \text { odd }} 4^{s} \sigma(m-1, s, \nu)\left(k V_{1}^{j} V_{2}^{k-1} T^{l+s+1} q^{m-1-s}\right. \\
& \left.+V_{1}^{j+1} V_{2}^{k} T^{l+s} q^{m-1-s}+V_{1}^{j} V_{2}^{k+1} T^{l+s} q^{m-1-s}\right) \\
& -2 \mu \sum_{s=0, s \text { even }} 4^{s}\left(\begin{array}{c}
m \\
s+1
\end{array}\right)\left(k V_{1}^{j} V_{2}^{k-1} T^{l+s+1} q^{m-1-s}\right. \\
& \left.+V_{1}^{j+1} V_{2}^{k} T^{l+s} q^{m-1-s}+V_{1}^{j} V_{2}^{k+1} T^{l+s} q^{m-1-s}\right) \\
& -2 \mu \sum_{s=0, s \text { odd }} 4^{s}\left(\begin{array}{c}
m \\
s+1
\end{array}\right)\left(k V_{1}^{j} V_{2}^{k-1} T^{l+s+1} q^{m-1-s}\right. \\
& \left.+V_{1}^{j+1} V_{2}^{k} T^{l+s} q^{m-1-s}-V_{1}^{j} V_{2}^{k+1} T^{l+s} q^{m-1-s}\right) .
\end{aligned}
$$

To compute the action of $X_{1}^{\prime}$ we shall use that

$$
\Phi^{(s)}\left(X_{1}^{\prime}\right)=\left\{\begin{array}{l}
Y_{1} M^{11} \otimes 1=-i \mu Y_{1}, \text { if } s \text { is even, } \\
i Y_{1}^{\prime} M^{11} \otimes 1=\mu Y_{1}^{\prime}, \text { if } s \text { is odd }
\end{array}\right.
$$

Therefore we get

$$
\begin{aligned}
X_{1}^{\prime} & \cdot V_{1}^{j} V_{2}^{k} T^{l} q^{m} \otimes 1 \\
= & i j\left(\frac{\nu+\mu}{2}+k-l-j-m+1\right) V_{1}^{j-1} V_{2}^{k} T^{l} q^{m} \otimes 1 \\
& -i k\left(\frac{\mu-\nu}{2}+k+m-1\right) V_{1}^{j} V_{2}^{k-1} T^{l} q^{m} \otimes 1 \\
& +i l V_{1}^{j+1} V_{2}^{k} T^{l-1} q^{m} \otimes 1-i l V_{1}^{j} V_{2}^{k+1} T^{l-1} q^{m} \otimes 1 \\
& -2 i \sum_{s=0, s \text { even }} 4^{s} \sigma(m-1, s, \nu)\left(k V_{1}^{j} V_{2}^{k-1} T^{l+s+1} q^{m-1-s}\right. \\
& \left.+V_{1}^{j+1} V_{2}^{k} T^{l+s} q^{m-1-s}+V_{1}^{j} V_{2}^{k+1} T^{l+s} q^{m-1-s}\right) \\
& +2 i \sum_{s=0, s \text { odd }} 4^{s} \sigma(m-1, s, \nu)\left(k V_{1}^{j} V_{2}^{k-1} T^{l+s+1} q^{m-1-s}\right. \\
& \left.+V_{1}^{j+1} V_{2}^{k} T^{l+s} q^{m-1-s}-V_{1}^{j} V_{2}^{k+1} T^{l+s} q^{m-1-s}\right) \\
& +2 i \mu \sum_{s=0, s \text { even }} 4^{s}\left(\begin{array}{c}
m+1 \\
s+1
\end{array}\right)\left(k V_{1}^{j} V_{2}^{k-1} T^{l+s+1} q^{m-1-s}\right. \\
& \left.+V_{1}^{j+1} V_{2}^{k} T^{l+s} q^{m-1-s}-V_{1}^{j} V_{2}^{k+1} T^{l+s} q^{m-1-s}\right) \\
& +2 i \mu \sum_{s=0, s \text { odd }} 4^{s}\left(\begin{array}{c}
m \\
s+1
\end{array}\right)\left(k V_{1}^{j} V_{2}^{k-1} T^{l+s+1} q^{m-1-s}\right.
\end{aligned}
$$




$$
\left.+V_{1}^{j+1} V_{2}^{k} T^{l+s} q^{m-1-s}+V_{1}^{j} V_{2}^{k+1} T^{l+s} q^{m-1-s}\right)
$$

Thus

$$
\begin{aligned}
& \left(X_{1}-i X_{1}^{\prime}\right) \cdot V_{1}^{j} V_{2}^{k} T^{l} q^{m} \otimes 1 \\
& =j(\nu+\mu+2 k-2 l-2 j-2 m+2) V_{1}^{j-1} V_{2}^{k} T^{l} q^{m} \otimes 1 \\
& \quad-2 l V_{1}^{j} V_{2}^{k+1} T^{l-1} q^{m} \otimes 1-4 \sum_{s=0}^{m-1} 4^{s} \sigma(m-1, s, \nu) V_{1}^{j} V_{2}^{k+1} T^{l+s} q^{m-1-s} \\
& \quad-4 \mu \sum_{s=0}^{m-1}(-1)^{s} 4^{s}\left(\begin{array}{c}
m \\
s+1
\end{array}\right) V_{1}^{j} V_{2}^{k+1} T^{l+s} q^{m-1-s} \otimes 1,
\end{aligned}
$$

and

$$
\begin{aligned}
& \left(X_{1}+i X_{1}^{\prime}\right) \cdot V_{1}^{j} V_{2}^{k} T^{l} q^{m} \otimes 1= \\
& k(\mu-\nu+2 k+2 m-2) V_{1}^{j} V_{2}^{k-1} T^{l} q^{m} \otimes 1-2 l V_{1}^{j+1} V_{2}^{k} T^{l-1} q^{m} \otimes 1 \\
& +4 k \sum_{s=0}^{m-1}(-1)^{s} 4^{s} \sigma(m-1, s, \nu) V_{1}^{j} V_{2}^{k-1} T^{l+s+1} q^{m-1-s} \\
& +4 \sum_{s=0}^{m-1}(-1)^{s} 4^{s} \sigma(m-1, s, \nu) V_{1}^{j+1} V_{2}^{k} T^{l+s} q^{m-1-s} \\
& \quad-4 \mu k \sum_{s=0}^{m-1} 4^{s}\left(\begin{array}{c}
m \\
s+1
\end{array}\right) V_{1}^{j} V_{2}^{k-1} T^{l+s+1} q^{m-1-s} \otimes 1 \\
& -4 \mu \sum_{s=0}^{m-1} 4^{s}\left(\begin{array}{c}
m \\
s+1
\end{array}\right) V_{1}^{j+1} V_{2}^{k} T^{l+s} q^{m-1-s} \otimes 1 .
\end{aligned}
$$

Proposition 3.4. The coefficients $a_{j, k, l, m}(\nu+\rho, \lambda)$ for the $\chi$-Whittaker $u(\lambda$, $\nu+\rho)$ in $M(\nu+\rho)$ satisfy the following recurrence relations:

$$
\begin{array}{r}
\lambda a_{j, k, l, m}=(j+1)(\nu+\mu+2 k-2 l-2 m) a_{j+1, k, l, m}-2(l+1) a_{j, k-1, l+1, m} \\
-4 \sum_{s=0}^{l} 4^{s}\left(\sigma(m+s, s, \nu)+\mu(-1)^{s}\left(\begin{array}{c}
m+1+s \\
s+1
\end{array}\right)\right) a_{j, k-1, l-s, m+1+s}
\end{array}
$$


(3.31) $\lambda a_{j, k, l, m}=(k+1)(\mu-\nu+2 k+2 m) a_{j, k+1, l, m}-2(l+1) a_{j-1, k, l+1, m}$ $+4(k+1) \sum_{s=0}^{l-1} 4^{s}\left((-1)^{s} \sigma(m+s, s, \nu)-\mu\left(\begin{array}{c}m+1+s \\ s+1\end{array}\right)\right) a_{j, k+1, l-s-1, m+1+s}$ $+4 \sum_{s=0}^{l} 4^{s}\left((-1)^{s} \sigma(m+s, s, \nu)-\mu\left(\begin{array}{c}m+1+s \\ s+1\end{array}\right)\right) a_{j-1, k, l-s, m+1+s}$

$$
\begin{aligned}
& (j+1)(k+1) a_{j+1, k+1, l, m}=(l+1) a_{j, k, l+1, m} \\
& +2 \sum_{s=0}^{l} 4^{s}\left(\sigma(m+s, s, \nu)+\mu(-1)^{s}\left(\begin{array}{c}
m+1+s \\
s+1
\end{array}\right)\right) a_{j, k, l-s, m+1+s}
\end{aligned}
$$

$$
\begin{gathered}
(l+1) a_{j, k, l+1, m}=2 \sum_{s=0}^{l} 4^{s}\left((-1)^{s} \sigma(m+s, s, \nu)\right. \\
\left.-\mu\left(\begin{array}{c}
m+s+1 \\
s+1
\end{array}\right)\right) a_{j, k, l-s, m+1+s .}
\end{gathered}
$$

Proof. The equations follow from the definition of the Whittaker vector, Formulas (3.24), (3.25), (3.28) and (3.29). We note that if we set $\mu=0$ we get the formulas given in $[\mathbf{K b}]$, Proposition 3.5.

Equation (3.33) implies that $a_{j, k, l, m}=b_{j, k, l, m} a_{j, k, 0, l+m}$, where $b_{j, k, l, m}$ satisfies the formulas:

$$
\begin{aligned}
& b_{j, k, 0, m}= 1 \\
&(l+1) b_{j, k, l, m}=2 \sum_{s=0}^{l} 4^{s}\left((-1)^{s} \sigma(m+s, s, \nu)\right. \\
&\left.\quad-\mu\left(\begin{array}{c}
m+s+1 \\
s+1
\end{array}\right)\right) b_{j, k, l-s, m+1+s .} .
\end{aligned}
$$

From (3.34) and (3.35) we conclude that $b_{j, k, l, m}$ doesn't depend on $j$ and $k$. Therefore we shall use the notation $b_{l, m}$ instead of $b_{j, k, l, m}$.

Formulas (3.30) and (3.32) imply that

$$
\lambda a_{j, k, l, m}=(j+1)(\nu+\mu-2 l-2 m) a_{j+1, k, l, m},
$$

hence

$$
a_{j, k, l, m}=\frac{\lambda^{j}}{j ! \prod_{i=l+m}^{j+l+m-1}(\nu+\mu-2 i)} a_{0, k, l, m}
$$


From Formulas (3.31) and (3.33) we get

$$
\lambda a_{j, k, l, m}=(k+1)(\mu-\nu+2 k+2 m+2 l) a_{j, k, l, m},
$$

which implies

$$
a_{j, k, l, m}=\frac{(-1)^{k} \lambda^{k}}{k ! \prod_{i=l+m}^{k+l+m}(\nu-\mu-2 i)} a_{j, 0, l, m} .
$$

Finally, (3.37) together with (3.39) implies

$$
a_{j, k, l, m}=\frac{(-1)^{k} \lambda^{j+k}}{j ! k ! \prod_{i=l+m}^{j+l+m-1}(\nu+\mu-2 i) \prod_{i=l+m}^{k+l+m}(\nu-\mu-2 i)} a_{0,0, l, m} .
$$

If we set $l=0$ in the Formulas (3.32) and (3.33) we get:

$$
(j+1)(k+1) a_{j+1, k+1,0, m}=4 \sigma(m, 0, \nu) a_{j, k, 0, m+1} .
$$

But $\sigma(m, 0, \nu)=\sum_{j=0}^{m}(n+\nu-2(m-j))=(m+1)(n+\nu-m)$ and this together with (3.36) and (3.38) imply

$$
a_{j, k, 0, m+1}=\frac{-\lambda^{2}}{4(m+1)(n+\nu-m)(\nu+\mu-2 m)(\nu-\mu-2 k-2 m)} a_{j, k, 0, m}
$$

and then

$$
a_{j, k, 0, m}=\frac{(-1)^{m} \lambda^{2 m}}{4^{m} m ! \prod_{i=0}^{m-1}(n+\nu-i) \prod_{i=j}^{j+m-1}(\nu+\mu-2 i) \prod_{i=k}^{k+m-1}(\nu-\mu-2 i)} a_{j, k, 0,0} .
$$

Finally, we obtain the following explicit formula for the coefficient $a_{j, k, l, m}$ :

$$
\begin{aligned}
& a_{j, k, l, m}(\nu, \lambda) \\
& =b_{l, m}(\nu) a_{j, k, 0, l+m}(\nu, \lambda) \\
& =\frac{b_{l, m}(\nu)(-1)^{k+l+m} \lambda^{j+k+2 l+2 m}}{j ! k !(l+m) ! 4^{l+m} \prod_{i=0}^{j+l+m-1}(\nu+\mu-2 i) \prod_{i=0}^{k+l+m-1}(\nu-\mu-2 i) \prod_{i=0}^{l+m-1}(n+\nu-i)} a_{0,0,0,0} .
\end{aligned}
$$

Now let $M(-\nu)$ be the Verma module $\mathcal{U}(\mathfrak{g}) \otimes_{\mathcal{U}(\mathfrak{p})} \mathbb{C}_{-\nu-\rho}$, where $\mathbb{C}_{-\nu-\rho}$ is the $\mathfrak{p}$-module with $\mathfrak{m}$ acting by $d \xi_{\mu}, \mathfrak{n}$ acting by 0 and $a$ acting by $-\nu-\rho$. 
Let $u(\lambda,-\nu)=\sum_{I} a_{I}(\lambda, \nu) Y(I) \otimes 1$ be the $\chi$-Whittaker vector on the Verma module $M(-\nu)$, with $\chi$ as in Formula (2.4).

To obtain an explicit formula for the Whittaker vector $u(\lambda,-\nu)$ we change the parameter $\nu$ in Formulas (3.10) and (3.43) into $-\nu-\rho$, since $H$ acts by $-\nu(H)-\rho(H)$. Recall that for $G \simeq \mathrm{SU}(n+1,1)$ we have $\rho(H)=n+1$. With this parametrization and setting $a_{0,0,0}=1$ in the case $n=1$ and $a_{0,0,0,0}=1$ in the case $n>1$ we get

$$
a_{j, k, l}(\lambda,-\nu-\rho)=\frac{(-1)^{j+l}(\lambda / 2)^{j+k+2 l} \prod_{i=k+l+1}^{j+k+l}(\nu+i)}{j ! k ! l ! \prod_{i=1}^{j+l}\left(\frac{\nu-\mu}{2}+i\right) \prod_{i=1}^{k}\left(\frac{\nu+\mu}{2}+i\right) \prod_{i=1}^{j+l}(\nu+i)}
$$

$$
=\frac{b_{l, m}(-\nu-\rho)(-1)^{j} \lambda^{j+k+l, m}(\lambda,-\nu-\rho)}{j ! k !(l+m) ! 4^{l+m} \prod_{i=1}^{j+l+m}\left(\frac{\nu-\mu+n}{2}+i\right) \prod_{i=1}^{k+l+m}\left(\frac{\nu+\mu+n}{2}+i\right) \prod_{i=1}^{l+m}(\nu+i)} .
$$

Furthermore, it will be convenient to multiply $u(\lambda,-\nu-\rho)$ by the normalizing factor $I(\nu)=\left[\Gamma\left(\frac{\nu-\mu+n}{2}\right)\right]^{-1}\left[\Gamma\left(\frac{\nu+\mu+n}{2}\right)\right]^{-1}[\Gamma(\nu+1)]^{-1}$ in order to obtain a holomorphic Whittaker vector $\widetilde{u}(\lambda,-\nu-\rho)$. We may now state the main result in this section:

Theorem 3.5. Let $G$ be locally isomorphic with $\mathrm{SU}(n+1,1)$. Then a holomorphic $\chi$-Whittaker vector in $M(-\nu)$ is given by:

(1) If $n=1$ then

$$
\begin{aligned}
& \widetilde{u}(\lambda,-\nu-\rho) \\
& =\sum_{j, k, l \geq 0} \frac{(-1)^{j+l}(\lambda / 2)^{j+k+2 l} \prod_{i=k+l+1}^{j+k+l}(\nu+i) V_{1}^{j} V_{2}^{k} T^{l}}{j ! k ! l ! \Gamma\left(\frac{\nu-\mu}{2}+j+l+1\right) \Gamma\left(\frac{\nu+\mu}{2}+k+1\right) \Gamma(\nu+j+l+1)},
\end{aligned}
$$


(2) if $n>1$ then

$$
\begin{aligned}
& (3.47) \quad \widetilde{u}(\lambda,-\nu-\rho)=\sum_{j, k, l, m \geq 0} \\
& \cdot \frac{(-1)^{j} b_{l, m}(-\nu-\rho) \lambda^{j+k+2 l+2 m} V_{1}^{j} V_{2}^{k} T^{l} q^{m}[\Gamma(\nu+l+m+1)]^{-1}}{j ! k !(l+m) ! 4^{l+m} \Gamma\left(\frac{\nu-\mu+n}{2}+j+l+m+1\right) \Gamma\left(\frac{\nu+\mu+n}{2}+k+l+m+1\right)} .
\end{aligned}
$$

Remark 1. We observe from the definition of $\sigma(m, s, \nu)$ that

$$
\begin{aligned}
& \sigma(m+s, s,-\nu-\rho) \pm \mu\left(\begin{array}{c}
m+1+s \\
s+1
\end{array}\right) \\
& =\left(\begin{array}{c}
m+1+s \\
s
\end{array}\right)(m+1)\left(\frac{-\nu-1-2 m \pm \mu}{s+1}\right)+\frac{2 m}{s+2} .
\end{aligned}
$$

This together with (3.35) imply that $b_{l, m}(-\nu-\rho)$ is a polinomial in $\nu$ of degree $l$.

\section{An explicit formula for the $\tau$-function.}

Let $G$ be a Lie group locally isomorphic to $\mathrm{SU}(n+1,1)$. We recall from $\S 2$ the definition of the $\tau$-function:

$$
\tau\left(\chi_{1}, \chi, u a, \nu\right)=\sum_{I \in \mathbb{N}} a_{I}(-\nu) d \chi_{1}\left(\operatorname{Ad}(u a s)^{-1} Y(I)^{T}\right)
$$

where $u \in M, a \in A$ and $u(\lambda,-\nu)=\sum_{I} a_{I}(\lambda,-\nu) Y(I) \otimes 1$ is the $\chi$ Whittaker vector on the Verma module $M(-\nu)$. This function appears in the $\chi_{1}$-Fourier coefficient $D_{\chi_{1}}^{\chi}$ of the Poincaré series studied in $[\mathbf{M W}]$ and in the integral kernel of the Kuznetsov type formula in [MW1]. The case $\chi=\chi_{1}$ has special interest because the poles of the meromorphic continuation of $D_{\chi}^{\chi}(P, P, \nu)$ to $\mathbb{C}$ lie exactly at spectral parameters, that is, the nonzero eigenvalues of the Casimir operator $C$ on $L_{d}^{2}(\Gamma \backslash G / K)$ have the form $\nu_{j}(H)^{2}-$ $\rho(H)^{2}$, where $\nu_{j}$ is a pole of $\left\{D_{\chi}^{\chi}(P, P, \nu) \mid \chi \in\left(\Gamma_{N} \backslash N\right)^{\wedge}-1\right\}$ in the closed right half plane. Our main goal in this section will be to give an explicit formula for $\tau(\chi, \chi, u a, \nu)$.

For this purpose we need to compute $\operatorname{Ad}\left(u a s^{*}\right)^{-1} Y(I)$. If we take $s^{*}=$ $\left(\begin{array}{ccc}0 & 0 & i \\ 0 & I_{n} & 0 \\ i & 0 & 0\end{array}\right)$, where $I_{n}$ denotes the $n \times n$ identity matrix, we have that $\operatorname{Ad}\left(s^{*}\right)^{-1} Y_{i}$ $=X_{i}{ }^{\prime}, \operatorname{Ad}\left(s^{*}\right)^{-1} Y_{i}{ }^{\prime}=-X_{i}$, and therefore $\operatorname{Ad}\left(s^{*}\right)^{-1} V_{1}=1 / 2\left(X_{1}{ }^{\prime}-i X_{1}\right)$ and $\operatorname{Ad}\left(s^{*}\right)^{-1} V_{2}=1 / 2\left(-X_{1}{ }^{\prime}-i X_{1}\right)$. Also, since

$$
\operatorname{Ad}\left(u^{-1}\right) V_{1}=c_{1} V_{1}+d_{1} V_{2}+\sum_{j=2}^{n}\left(c_{j} Y_{j}+d_{j} Y_{j}^{\prime}\right)
$$


for some coefficients $c_{j}$ and $d_{j}$ and using the fact that $\left\langle V_{1}, V_{1}\right\rangle=\left\langle V_{2}, V_{2}\right\rangle=$ $1 / 2$, we may write

$$
\operatorname{Ad}\left(u^{-1}\right) V_{1}=2\left\langle V_{1}, \operatorname{Ad}(u) V_{1}\right\rangle V_{1}+2\left\langle V_{1}, \operatorname{Ad}(u) V_{2}\right\rangle V_{2}+\sum_{j=2}^{n}\left(c_{j} Y_{j}+d_{j} Y^{\prime j}\right) .
$$

In the same way we obtain

$$
\operatorname{Ad}\left(u^{-1}\right) V_{2}=2\left\langle V_{2}, \operatorname{Ad}(u) V_{1}\right\rangle V_{1}+2\left\langle V_{2}, \operatorname{Ad}(u) V_{2}\right\rangle V_{2}+\sum_{j=2}^{n}\left(\widetilde{c}_{j} Y_{j}+\widetilde{d}_{j} Y_{j}^{\prime}\right) .
$$

As $d \chi\left(\operatorname{Ad}\left(s^{*}\right)^{-1} V_{1}\right)=d \chi\left(\operatorname{Ad}\left(s^{*}\right)^{-1} V_{2}\right)=\frac{-i \lambda}{2}$ we get the formula

$$
\begin{aligned}
& d \chi\left(\operatorname{Ad}\left(u_{a s^{*}}\right)^{-1} V_{1}{ }^{j} V_{2}{ }^{k}\right) \\
& =a^{(j+k) \alpha}\left(\frac{-i \lambda}{2}\right)^{j+k} 2^{j+k}\left\langle V_{1}, \operatorname{Ad}(u)\left(V_{1}+V_{2}\right)\right\rangle^{j}\left\langle V_{2}, \operatorname{Ad}(u)\left(V_{1}+V_{2}\right)\right\rangle^{k} \\
& =\left(-i \lambda a^{\alpha}\right)^{j+k}\left\langle V_{1}, \operatorname{Ad}(u)\left(V_{1}+V_{2}\right)\right\rangle^{j}\left\langle V_{2}, \operatorname{Ad}(u)\left(V_{1}+V_{2}\right)\right\rangle^{k} .
\end{aligned}
$$

In the case of $\mathrm{SU}(2,1)$ the last formula can be simplified. If we take $u=u_{\theta}=\left(\begin{array}{ccc}e^{i \theta} & 0 & 0 \\ 0 & e^{-2 i \theta} & 0 \\ 0 & 0 & e^{i \theta}\end{array}\right)$, then $\operatorname{Ad}(u) V_{1}=e^{3 i \theta} V_{1}, \operatorname{Ad}(u) V_{2}=e^{-3 i \theta} V_{2}$ and so

$$
\begin{aligned}
& d \chi\left(\operatorname{Ad}\left(u a s^{*}\right)^{-1} V_{1}^{j} V_{2}^{k}\right) \\
& =\left(-i \lambda a^{\alpha}\right)^{j+k} e^{3 i(j-k) \theta}\left\langle V_{1}, V_{1}\right\rangle^{j}\left\langle V_{2}, V_{2}\right\rangle^{k}=\left(\frac{-i \lambda a^{\alpha}}{2}\right)^{j+k} e^{3 i(j-k) \theta} .
\end{aligned}
$$

Now, $T$ is $M$-invariant and it belongs to $\mathfrak{g}_{-2 \alpha}$. Then $d \chi\left(\operatorname{Ad}\left(s^{*}\right)^{-1} T^{l}\right)=0$ if $l>0$. Also, from the computations above we get:

$$
d \chi\left(\operatorname{Ad}\left(u a s^{*}\right)^{-1} q^{m}\right)=a^{2 m \alpha} d \chi\left(X_{1}^{2}\right)^{m}=\left(\lambda a^{\alpha}\right)^{2 m} .
$$

We shall use the following notation: Let be $\lambda \in i \mathbb{R}, a \in A$ and $u \in M$. We shall write

$$
\begin{aligned}
z & =z(\lambda, a)=-i(\lambda / 2)^{2} a^{\alpha}, \\
\omega_{i} & =\omega_{i}(u)=\left\langle V_{i}, \operatorname{Ad}(u)\left(V_{1}+V_{2}\right)\right\rangle, i=1,2 .
\end{aligned}
$$

We note that $z \in i \mathbb{R}^{>0}$, thus $\bar{z}=-z$. We also recall the definition of the generalized hypergeometric functions. We shall follow the notation of [Sl]. Let $(a)=\left(a_{1}, a_{2}, \ldots, a_{p}\right)$ and $(b)=\left(b_{1}, \ldots, b_{q}\right)$. The generalized hypergeometric function ${ }_{p} F_{q}((a) ;(b) ; y)$ is defined as follows:

$$
{ }_{p} F_{q}((a) ;(b) ; y)=\sum_{n=0}^{\infty} \frac{\left(a_{1}\right)_{n} \ldots\left(a_{p}\right)_{n}}{n !\left(b_{1}\right)_{n} \ldots\left(b_{q}\right)_{n}} y^{n}
$$


where $(c)_{n}=c(c+1) \ldots(c+n-1)=\frac{\Gamma(c+n)}{\Gamma(c)}$ if $n \geq 1$ and $(c)_{0}=1$. We recall that if $p \leq q$ then ${ }_{p} F_{q}((a) ;(b) ; y)$ converges for every finite value of $y$. For $p=0$ and $q=1$ then ${ }_{0} F_{1}$ corresponds to the classical Bessel function $I_{\nu}$, that is

$$
{ }_{0} F_{1}(; b ; z)=\Gamma(b)(z)^{b-1} I_{b-1}\left(2 z^{\frac{1}{2}}\right) .
$$

We now state the main result of this work.

Theorem 4.1. Let $G$ be isomorphic to $\mathrm{SU}(n+1,1)$, and let $\tau(\chi, \chi, u a, \nu)$ be as in (4.1), $u \in M, a \in A$ and $\chi$ a character on $N$ as in (2.4). Let $z, \omega_{1}$ and $\omega_{2}$ be as in (4.4) and (4.5). If $n=1$ let $u=u_{\theta}$ as in (4.3). Then

$\tau\left(\chi, \chi, u_{\theta}, \nu\right)$

$$
\begin{aligned}
& =\sum_{j, k \geq 0} \frac{(-1)^{j} \prod_{s=1}^{j+k}(\nu+s) z^{j+k} e^{3 i(j-k) \theta}}{j ! k ! \prod_{s=1}^{j}(\nu+s)\left(\frac{\nu-\mu}{2}+s\right) \prod_{s=1}^{k}(\nu+s)\left(\frac{\nu+\mu}{2}+s\right)} \\
& =\sum_{j \geq 0} \frac{\left(\overline{z e^{-3 i \theta}}\right)^{j}}{j !\left(\frac{\nu-\mu}{2}+1\right)_{j}}{ }_{1} F_{2}\left(\nu+j+1 ; \nu+1, \frac{\nu+\mu}{2}+1, z e^{-3 i \theta}\right) \\
& =\sum_{m \geq 0}(\nu+1)_{m} \sum_{j=0}^{m} \frac{\left(\overline{z e^{-3 i \theta}}\right)^{j}\left(z e^{3 i \theta}\right)^{m-j}}{j !(m-j) !(\nu+1)_{j}\left(\frac{\nu-\mu}{2}+1\right)_{j}(\nu+1)_{m-j}\left(\frac{\nu+\mu}{2}+1\right)_{m-j}} .
\end{aligned}
$$

If $n>1$ then

$\tau(\chi, \chi, u a, \nu)$

$$
=\sum_{j, k, m \geq 0} \frac{(-1)^{k}(\bar{z} / 2)^{j+k+2 m} \omega_{1}^{j} \omega_{2}^{k}}{j ! k ! m ! \prod_{s=1}^{j+m}\left(\frac{\nu-\mu+n-1}{2}+s\right) \prod_{s=1}^{k+m}\left(\frac{\nu+\mu+n-1}{2}+s\right) \prod_{s=1}^{m}(\nu+s)}
$$

$$
\begin{aligned}
& =\sum_{m \geq 0} \frac{(z / 2)^{2 m}}{m !(\nu+1)_{m}\left(\frac{\nu+\mu+n+1}{2}\right)_{m}\left(\frac{\nu-\mu+n+1}{2}\right)_{m}} \\
& \quad \cdot{ }_{0} F_{1}\left(\frac{\nu-\mu+1}{2}+m+1, \frac{\bar{z} \omega_{1}}{2}\right)_{0} F_{1}\left(\frac{\nu+\mu+1}{2}+m+1, \frac{z \omega_{2}}{2}\right)
\end{aligned}
$$




$$
\begin{aligned}
= & \Gamma\left(\frac{\nu-\mu+n+1}{2}\right) \Gamma\left(\frac{\nu+\mu+n+1}{2}\right)\left(\frac{\bar{z} \omega_{1}}{2}\right)^{\frac{\nu-\mu+n+1}{2}}\left(\frac{z \omega_{2}}{2}\right)^{\frac{\nu+\mu+n+1}{2}} \\
& \cdot \sum_{m \geq 0} \frac{(\bar{z} / 2)^{2 m}\left(\frac{\bar{z} \omega_{1}}{2}\right)^{m}\left(\frac{z \omega_{2}}{2}\right)^{m}}{m !(\nu+1)_{m}} I_{\frac{\nu-\mu+n-1}{2}+m}\left(\left(2 \bar{z} \omega_{1}\right)^{1 / 2}\right) I_{\frac{\nu+\mu+n-1}{2}+m}\left(\left(2 z \omega_{2}\right)^{1 / 2}\right) .
\end{aligned}
$$

The first expressions for the $\tau$-functions are easily obtained from the definition of the $\tau$-function and the formulas in (4.2) and (4.3). Reordering these series and using the definition of the hypergeometric functions one gets the other alternative formulas.

Remark 2. The formula for the $\tau$-function in (4.6) suggests that if $\mu \in \mathbb{N}$ is fixed, then there are a finite number of possible poles of the $\tau$ function in $R e \nu>0$. In particular, for $\mathrm{SU}(2,1)$ there should be simple poles at $\nu=\mu-2 t, t \in \mathbb{N}$, provided that $\mu-2 t>0$. We shall prove that these poles really exist by computing the residue at the point and showing that it is not zero.

Let $\mu \in \mathbb{N}$, and let $\nu \in \mathfrak{a}^{*}, \nu>\rho$ such that $\nu=\mu-2 t$ for some $t \in \mathbb{N}$. Note that as $\lambda \in i \mathbb{R}$ we have that $-i(\lambda / 2)^{2} a^{\alpha}=i x$, for some $x \in \mathbb{R}^{>0}$. Thus

$$
\begin{aligned}
& \operatorname{Res}_{\nu=\nu_{t}} \tau(\chi, \chi, u a, \nu) \\
& =\lim _{\nu \rightarrow \nu_{t}}\left(\nu-\nu_{t}\right) \tau(\chi, \chi, u a, \nu) \\
& =\lim _{\nu \rightarrow \nu_{t}}\left(\nu-\nu_{t}\right) \sum_{\substack{0 \leq j<t \\
k \geq 0}} \frac{(-1)^{j} \prod_{s=1}^{k}(\nu+j+s)(i x)^{j+k} e^{3 i(j-k) \theta}}{j}\left(\frac{\nu-\mu}{2}+s\right) \prod_{s=1}^{k}(\nu+s)\left(\frac{\nu+\mu}{2}+s\right) \\
& +\lim _{\nu \rightarrow \nu_{t}}\left(\nu-\nu_{t}\right) \sum_{\substack{j \geq t \\
k \geq 0}} \frac{(-1)^{j} \prod_{s=1}^{k}\left(\nu+j+s ! \prod_{s=1}^{j}\left(\frac{\nu-\mu}{2}+s\right) \prod_{s=1}^{k}(\nu+s)\left(\frac{\nu+\mu}{2}+s\right)\right.}{} .
\end{aligned}
$$


The first term in the last expression of the residue is zero because the infinite sum involved is holomorphic at $\nu=\nu_{t}$. Then

$$
\begin{aligned}
& \operatorname{Res}_{\nu=\nu_{t}} \tau(\chi, \chi, u a, \nu) \\
& =\lim _{\nu \rightarrow \nu_{t}} \sum_{\substack{j \geq t \\
k \geq 0}} \frac{2(-1)^{j} \prod_{s=1}^{k}(\nu+j+s)(i x)^{j+k} e^{3 i(j-k) \theta}}{j ! k ! \prod_{s=1}^{t-1}\left(\frac{\nu-\mu}{2}+s\right) \prod_{s=t+1}^{j}\left(\frac{\nu-\mu}{2}+s\right) \prod_{s=1}^{k}(\nu+s)\left(\frac{\nu+\mu}{2}+s\right)} .
\end{aligned}
$$

Putting $\theta=\pi / 6$ and using that $(-1)^{j}=i^{-2 j}$ we get

$$
(-1)^{j}(i x)^{j+k} e^{3 i(j-k) \theta}=i^{-j+k} x^{j+k} e^{i \pi / 2(j-k)}=x^{j+k}(-i)^{j-k} i^{j-k}=x^{j+k},
$$

which is a positive real number. Furthemore, the factor $\prod_{s=1}^{t-1}\left(\frac{\nu-\mu}{2}+s\right)$ does not depend on $j$ and $k$. Then

$$
\begin{aligned}
& \operatorname{Res}_{\nu=\nu_{t}} \tau(\chi, \chi, u(\pi / 6) a, \nu) \\
& =\frac{2}{\prod_{s=1}^{t-1}(-t+s)} \sum_{\substack{j \geq t \\
k \geq 0}} \frac{\prod_{s=1}^{k}\left(\nu_{t}+j+s\right) x^{j+k}}{\prod_{s=t+1}^{j}(-t+s) \prod_{s=1}^{k}\left(\nu_{t}+s\right)\left(\frac{\nu_{t}+\mu}{2}+s\right)} \\
& =(-1)^{t-1}(t-1) ! \sum_{\substack{j \geq t \\
k \geq 0}} \frac{\prod_{s=1}^{k}(\mu-2 t+j+s) x^{j+k}}{j !(j-t) ! \prod_{s=1}^{k}(\mu-2 t+s)(\mu+t+s)} .
\end{aligned}
$$

Now, the series involved in the last expression is convergent and the terms are strictly greater than zero. This shows that the residue of the $\tau$ function at $\nu=\nu_{t}$ is not zero.

This proves the existence of simple poles of the $\tau$ function for $\mathrm{SU}(2,1)$ in $\{\operatorname{Re} \nu>0\}$ at the real points $\mu-2, \mu-4, \ldots$ The same should be true for $\mathrm{SU}(n+1,1)$, for $n>1$ but we have not carried out the verification.

\section{References}

[BM] R.W. Bruggeman and R.J. Miatello, Estimates of Kloosterman sums for groups of real rank one, Duke Math. J., 80 (1995), 105-137, MR 98h:11060, Zbl 0866.11049.

[GW] R. Goodman and N.R. Wallach, Whittaker vectors and conical vectors, J. Funct. Anal., 39 (1980), 199-279, MR 82i:22018, Zbl 0475.22010. 
[Kb] N.P. Kisbye, The integral kernel in the Kuznetsov type sum formula for $\mathrm{SU}(n+$ 1, 1), Indag. Math., 12 (2001), 83-101, MR 2003g:22011.

[K] N.V.Kuznetsov, The Petersson hypothesis for parabolic forms of weight zero and the Linnik hypothesis. Sums of Kloosterman sums (Russian), Mat. Sbornik, 111(153) (1980), 334-383, MR 81m:10053, Zbl 0427.10016.

[MV] R.J. Miatello and J. Vargas, On $M_{\chi}$-invariants in $\mathcal{S}(\mathfrak{n})$ and $\mathcal{U}(\mathfrak{n})$, Comm. Alg., 23(6) (1995), 2027-2043, MR 96c:22025, Zbl 0829.22030.

[MW] R.J. Miatello and N.R. Wallach, Kuznetsov formulas for real rank one groups, J. Funct. Anal., 93 (1990), 171-206, MR 91h:11041, Zbl 0711.11023.

[MW1] _ Automorphic forms constructed from Whittaker vectors, J. Funct. Anal., 86 (1989), 411-487, MR 91b:11065, Zbl 0692.10029.

[Sl] L.J. Slater, Confluent Hypergeometric Functions, Cambridge University Press, 1960, MR 21 \#5753, Zbl 0086.27502.

Received October 10, 2002.

FAMAF, Universidad Nacional de Cordoba

Ciudad Universitaria

Medina Allende $\mathrm{S} / \mathrm{N}$

5000 CORDOBA

Argentina

E-mail address: kisbye@mate.uncor.edu 